\title{
Celebrating the 25th birthday of intracytoplasmic sperm injection
}

\author{
C L O'Neill and G D Palermo
}

The Ronald O. Perelman and Claudia Cohen Center for Reproductive Medicine, Weill Cornell Medicine, New York, New York, USA

Correspondence should be addressed to G D Palermo; Email: gdpalerm@med.cornell.edu

Reproduction (2017) 154 E3-E4

Among the different indications, once excluding combined or unexplained factors, causes of infertility can be equally attributed to each partner (CDC 2008). The introduction of in vitro insemination in the late 1970s was used to treat various forms of infertility related mainly to the female partner. However, soon after its inception, it was evident that there were limitations as proven by the inability of about $40 \%$ of the couples treated to achieve fertilization (Cohen et al. 1984).

Many different procedures ranging back since the 1980s have attempted to assist spermatozoa hindered by functional defects in successful penetration and fertilization of the oocyte, such as partial zona dissection (PZD) and sub-zonal injection (SUZI). The evolution of procedures attempting to manipulate both gametes, inspired by the micromanipulation performed in experimental animals eventually led to the alteration of the SUZI technique to the development of intracytoplasmic sperm injection (ICSI).

Although the injection of a single spermatozoon into the cytoplasm was first attempted in humans in 1988 (Lanzendorf et al. 1988), the first pregnancies were not reported until 1992 (Palermo et al. 1992), leading us to this year's celebration of the 25th anniversary of ICSI. This technique, in spite of a slow start and its controversial acceptance into practice due to the arbitrary selection of an individual spermatozoon, has proven to be consistently successful in cases with severe male factor infertility in providing clinical outcomes that are comparable to the ideal cases treated by standard in vitro insemination.

In this issue, we provide a historical overview that brought about the development of ICSI, described in the first chapter, The Pioneering of Intracytoplasmic Sperm Injection - Historical Perspectives (Rosenwaks \& Pereira 2017), delineating the progression of micromanipulation procedures leading up to the development of this celebrated technique. ICSI had been early attempted in experimental animals, albeit with slim success, until the first pregnancies reported in humans propelled this procedure to popular utilization (Palermo et al. 1992).
Indeed, ICSI is now performed in several other species as it will be described in the chapter of this issue dedicated to ICSI in Domestic and Wild Mammals (Salamone et al. 2017).

ICSI is now the leading treatment for male infertility, reliably achieving consistent fertilization and pregnancy with ejaculated or surgically retrieved spermatozoa whether utilized fresh or following cryopreservation. This result indicates that the release of a single spermatozoon directly into the ooplasm is able to overcome sperm component dysfunction and spermatogenic immaturity of the male gamete. The utilization and performance of ICSI in couples with severe male factor, together with some of the assays designed to provide specific information on the ideal spermatozoon to be selected for injection, are depicted in the chapter ICSI: State of the Art in Humans (Palermo et al. 2017).

In addition toalleviating various forms of maleinfertility, the inception of ICSI has been instrumental in facilitating the advance of other forms of reproductive techniques including the cryopreservation of oocytes, in vitro maturation and the increasing reliance on molecular assessment of single-gene defects in conceptuses via preimplantation genetic diagnosis (PGD).

Although it is now implemented with tens of thousands of cycles performed every year world-wide, qualms are still raised due to the fact that at times with ICSI, a morphologically suboptimal spermatozoon that has still retained viability is considered to be appropriate for injection. This generated concern regarding the health of the offspring. A survey of the literature including studies from our center on the well-being of offspring at different developmental ages until the assessment of the hormonal profile and its effect on the menstrual cycle of young ICSI women or spermatogenesis in young men generated from ICSI, will be discussed in the chapter The Safety of Intracytoplasmic Sperm Injection and Long-term Outcomes in relation to the procedure (Pereira et al. 2017).

Still, ICSI is limited by the presence of at least one fully formed spermatozoon with retained viability, 
therefore excluding treatment of patients with spermatogenic arrest or germ cell aplasia. Fortunately, recent achievements in regenerative medicine by the utilization of embryonic stem cells and more recently induced pluripotent stem cells (iPSCs) are focused on facilitating neogametogenesis. These advances may allow for eventual gamete production directly in vitro, via the aforementioned cells or the information gained from these studies may even guide to restoring in vitro the function of the germinal epithelium in patients with spermatogenic arrest. This field with its recent exciting accomplishments will be elucidated in this issue's chapter, Stem cells, in vitro gametogenesis, and male fertility (Nagamatsu \& Hayashi 2017).

The development of in vitro fertilization marks an important step in reproductive medicine, allowing childless couples to fulfill their dream. ICSI has further assisted couples characterized by various arrays of male reproductive dysfunction who were unable to achieve a pregnancy by standard in vitro fertilization. The power given to a single male gamete via this procedure by identifying, retrieving and injecting a single spermatozoon into the egg while maintaining its prominence as the ultimate treatment for male infertility has opened the door to the utilization of immature forms of the male gamete and together with upcoming new techniques in the field will allow us to treat men with complete spermatogenic failure.

In addition to this brief overview to this special issue, I invite all readers to watch the Video 1 'Editorial: 25 years of $\mathrm{ICSI}^{\prime}$, where the chapters and topics related to ICSI will be further introduced.

\section{Video 1}

25 years of ICSI. This is a brief video discussing the successes of intracytoplasmic sperm injection over the past quarter of a century since its inception and the trajectory of the role of the technique toward the future of assisted reproductive technology. View video from the online version of the article available at https://doi.org/10.1530/REP-17-0684.

\section{References}

CDC 2008 Assisted Reproductive Technology Success Rates. National Summary and Fertility Clinic Reports. National Center for Chronic Disease and Prevention and Health Promotion.

Cohen J, Fehilly C, Fishel S, Edwards R, Hewitt J, Rowland G, Steptoe P \& Webster J 1984 Male infertility successfully treated by in-vitro fertilisation. Lancet 323 1239-1240. (https://doi.org/10.1016/S01406736(84)91723-9)

Lanzendorf SE, Maloney MK, Veeck LL, Slusser J, Hodgen GD \& Rosenwaks Z 1988 A preclinical evaluation of pronuclear formation by microinjection of human spermatozoa into human oocytes. Fertility and Sterility 49 835-842. (https://doi.org/10.1016/S0015-0282(16)59893-8)

Nagamatsu G \& Hayashi K 2017 Stem cells, in vitro gametogenesis and male fertility. Reproduction 154 F79-F91. (https://doi.org/10.1530/REP17-0510)

Palermo G, Joris H, Devroey P \& Van Steirteghem AC 1992 Pregnancies after intracytoplasmic injection of single spermatozoon into an oocyte. Lancet 340 17-18. (https://doi.org/10.1016/0140-6736(92)92425-F)

Palermo GD, O'Neill CL, Chow S, Cheung S, Parella A, Pereira N \& Rosenwaks Z 2017 Intracytoplasmic sperm injection - State of the Art in Humans. Reproduction 154 F93-F110. (https://doi.org/10.1530/REP17-0092)

Pereira N, O'Neill C, Lu V, Rosenwaks Z \& Gianpiero DP 2017 The safety of intracytoplasmic sperm injection and long-term outcomes. Reproduction 154 F61-F70. (https://doi.org/10.1530/REP-17-0344)

Rosenwaks Z \& Pereira N 2017 The pioneering of intracytoplasmic sperm injection: historical perspectives. Reproduction 154 F71-F77. (https://doi.org/10.1530/REP-17-0308)

Salamone D, Canel NG \& Rodriguez MB 2017 Intracytoplasmic sperm injection in Domestic and Wild Mammals. Reproduction 154 F111-F124. (https://doi.org/10.1530/REP-17-0357)

Received 7 November 2017

First decision 20 November 2017

Revised manuscript received 20 November 2017

Accepted 4 December 2017 